\title{
Design and Evaluation of Korean Text Entry Methods for Mobile Phones
}

I vaylo I linkin

Department of Computer Science

Gettysburg College

Gettysburg, PA 17325 USA

ilinkin@gettysburg.edu

Sunghee Kim

Department of Computer Science

Gettysburg College

Gettysburg, PA 17325 USA

skim@gettysburg.edu

\author{
Abstract \\ This paper presents a new keypad layout for Korean \\ mobile phones and provides a preliminary evaluation of \\ the feasibility of the design. At present the keypad \\ layout for Korean mobile phones has not been \\ standardized and different manufacturers produce \\ phones with different layouts. The proposed layout is \\ inspired by the structure of the Korean script and is \\ designed to promote faster learning curve and ease of \\ use. \\ Keywords \\ Hangul, mobile phones, text entry, Fitt's mode

\section{General Terms} \\ Design, human factors

\section{ACM Classification Keywords} \\ H.5.2. User interfaces: Input devices and strategies

\section{I ntroduction} \\ The popularity and capabilities of mobile phones have \\ been developing at a staggering pace. They are used \\ for voice communication, email, digital planner, access \\ to news, maps and directions. The mobile phones have \\ created a cultural phenomenon by redefining \\ communication through the use of short messaging
}


service (SMS) - a means of communication via short text messages which is particularly popular in Europe and Asia.

As the use and capabilities of mobile phones increase, the need for intuitive, effective, and efficient mechanisms for interacting with the device and entering data is apparent. The challenge here is that the size of the device limits significantly the number of controls (keys) that it can accommodate. Moreover, increasing the number of controls does not necessarily imply efficient and effective interaction, since mobile phones are typically operated with one hand which restricts the range of motion.

Today the interface of a typical phone (the keypad) consists of 12 keys excluding the keys for initiating and concluding a phone call. The applications mentioned previously (email, SMS, planner) require a fair amount of text entry from the user. In general a language will have more letters (symbols) then the available keys on the keypad which implies that the arrangement of the letters on the keypad can affect significantly the user's experience with the phone.

This paper investigates keypad layout design for Korean text entry on mobile phones. The proposed layout is inspired by the structure of the Korean script which makes it possible to identify meaningful structural groups or basic elements from which the characters are built. It should be noted that the Korean script is alphabetic which makes designing a keypad layout a feasible task.

\section{Korean Writing System (Hangul)}

The Korean alphabet consists of 24 basic letters (14 consonants and 10 vowels) and 16 compound letters (5 consonants and 11 vowels):

\begin{tabular}{|c|c|}
\hline \multicolumn{2}{|r|}{ Consonants } \\
\hline basic & ᄀ ᄂ ᄃ こ 口 \\
\hline compound & $\pi$ 匹 \\
\hline \multicolumn{2}{|r|}{ Vowels } \\
\hline basic & 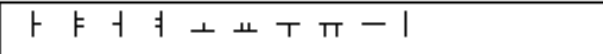 \\
\hline compound & 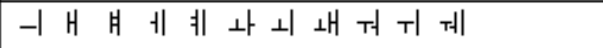 \\
\hline
\end{tabular}

Figure 1. The Korean alphabet.

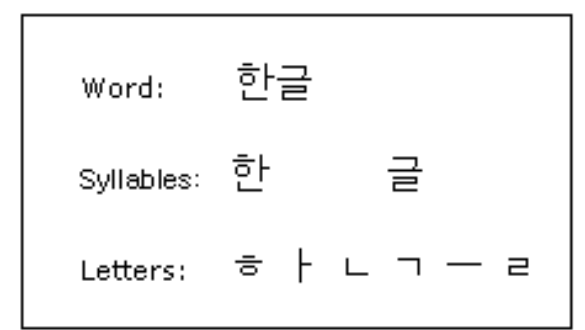

Figure 2. Example of Korean word and syllable structure.

A distinguishing characteristic of the Korean writing system is that the letters within a syllable are stacked according to predefined rules as shown in Figure 2.

Therefore, both the individual letters and the syllables can be treated as independent structural units. 


\section{Existing Text Entry Methods}

The predominant Korean text entry methods for mobile phones are Chon-ji-in and EZ-Hangul found on Samsung and LG phones, respectively.

Chon-ji-in Entry Method

Chon-ji-in (Figure 3) incorporates cultural elements to aid the user in adopting the technology. For example, the symbols corresponding to the number keys 1,2 , and 3 have the meaning sky $(\mid)$, ground $(-)$, and man $(\bullet)$, and all vowels are constructed using these keys (concepts). The consonants are assigned to the rest of the number keys and are produced by pressing the corresponding key until the desired letter is shown.

\begin{tabular}{|c|c|c|c|c|}
\hline 1 & 1 & 2 & • & - \\
\hline 4 & $\neg$ ᄀ & & $\llcorner$ ᄅ & $6 \sqsubset E$ \\
\hline 7 & ㅂㅍ & & ᄉ $\overline{0}$ & 9 万 \\
\hline * & & & $\begin{array}{ll}0 & 0\end{array}$ & $\#$ \\
\hline
\end{tabular}

Figure 3. Chon-ji-in keypad layout.

\begin{tabular}{|c|c|c|c|c|c|c|c|c|c|c|c|c|c|}
\hline \multirow{2}{*}{ 빨래 } & 7 & 7 & 7 & 1 & 2 & 5 & 5 & & 5 & 5 & 1 & 2 & 1 \\
\hline & \multicolumn{3}{|c|}{$\forall \forall$} & \multicolumn{2}{|c|}{ F } & \multicolumn{2}{|c|}{ 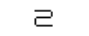 } & skip & \multicolumn{2}{|c|}{ ᄅ } & \multicolumn{3}{|c|}{$H$} \\
\hline
\end{tabular}

Figure 4. Example of Chon-ji-in key stroke sequence.

Chon-ji-in is easy to learn. However, in general it requires more key strokes for text entry.
EZ-Hangul Entry Method

EZ-Hangul (Figure 5) uses the concepts of adding a stroke and doubling to compose the letters based on their shapes. Only six consonants are assigned a number key and are used along with the control keys * (add a stroke) and \# (double) to compose the rest. The vowels are composed similarly.

Figure 6 shows a key stroke sequence for typing a compound consonant and a word using EZ-Hangul.

\begin{tabular}{|c|c|c|c|c|}
\hline 1 ר & 2 & \llcorner & & $1 \quad 1$ \\
\hline 4 ᄅ & 5 & 口 & 6 & $\perp \quad T$ \\
\hline$\curlywedge$ & 8 & 0 & 9 & I \\
\hline * add-stroke & 0 & - & $\#$ & double \\
\hline
\end{tabular}

Figure 5. EZ-Hangul keypad layout.

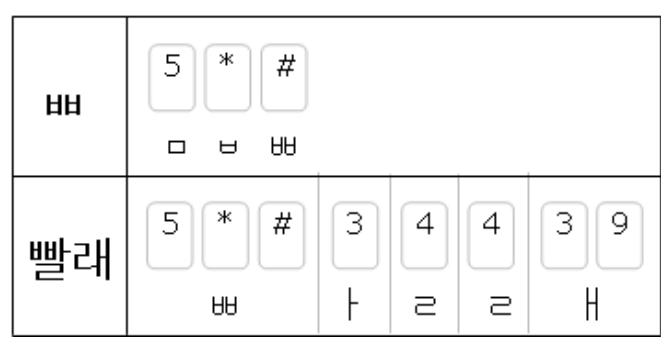

Figure 6. Example of EZ-Hangul key stroke sequence.

In general EZ-Hangul requires fewer key strokes. However, since only a subset of the letters is visible on the keypad and the composition rules are not readily apparent, it is considered somewhat difficult to learn. 


\section{Proposed Layout}

The design goals of the proposed layout (Figure 8) are:

1. Creating an intuitive layout that leads to faster learning curve and promotes recognition.

2. Minimizing the number of key strokes per letter.

To accomplish the first goal the design considers the shape of the Korean vowels to determine their placement on the keypad (Figure 7). The remaining seven keys are assigned to the consonants in an arrangement similar to Chon-ji-in.

The arrangement of the vowels also addresses the second goal since each vowel requires at most 3 strokes. Specifically, 5 vowels require 1 stroke, 10 require 2 strokes, and 6 require 3 strokes. In contrast using the Chon-ji-in text entry method 17 vowels require at least 3 key strokes, and using EZ-Hangul 9 vowels require at least 3 key strokes.

Figure 9 shows an example of the key strokes for typing a Korean word using the proposed layout.

\section{Evaluation}

Two standard metrics were used to evaluate the feasibility of the design: Key Strokes Per Character (KSPC) [7] and predicted movement time based on Fitt's model for two-dimensional tasks $[2,4,5,6]$. In addition, the three layouts were compared based on the finger travel distance required for text entry. The keypad dimensions used for Fitt's model and distance calculations are width $=9.5 \mathrm{~mm}$, height $=5 \mathrm{~mm}$, and gap $=2 \mathrm{~mm}$ as given in [4].

The input text used in the evaluation consists of 9248
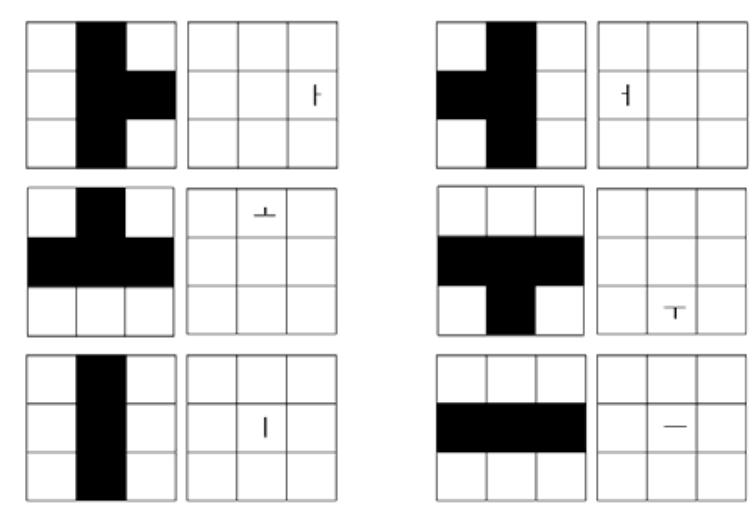

Figure 7. The intuition behind the placement of the vowels.

\begin{tabular}{|c|c|c|}
\hline 17 ר & $2+4$ & $3\llcorner ᄅ \square$ \\
\hline $4+\neq$ & $51--1$ & $6 \vdash k$ \\
\hline $7 \sqsubset E \widetilde{W}$ & $8 T \pi$ & $9 \forall$ 至 \\
\hline * $\curlywedge \wedge k$ & $\begin{array}{lll}0 & 0 & \overline{0}\end{array}$ & 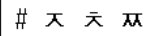 \\
\hline
\end{tabular}

Figure 8. The proposed layout.

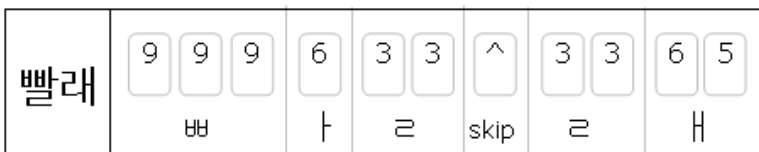

Figure 9. Example key stroke sequence using the proposed layout.

words, 250510 syllables, and 707220 letters [8].

The results in Figure 10 indicate that the performance of the proposed layout is comparable to that of Chon-jiin and EZ-Hangul. 


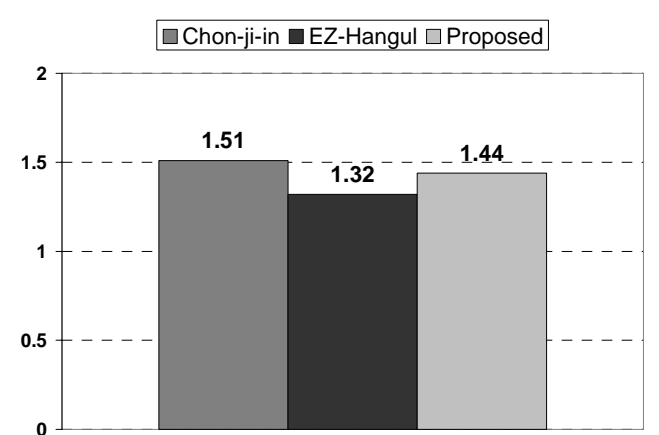

(a) Key Strokes Per Character

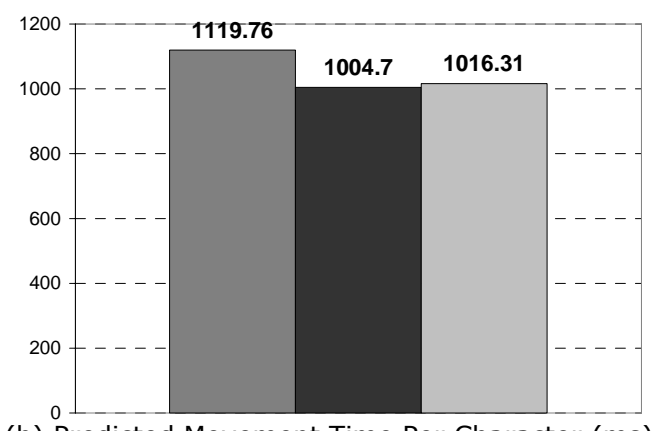

(b) Predicted Movement Time Per Character (ms)

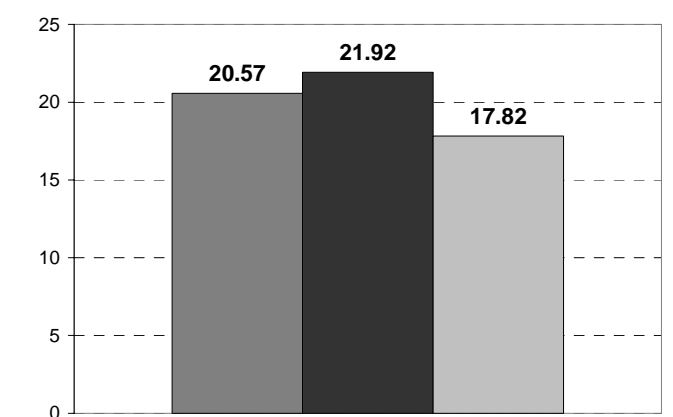

(c) Travel Distance Per Character (mm)

Figure 10. Evaluation results.
Key Strokes Per Character

The KSPC of the proposed layout is smaller than that of Chon-ji-in which is due to the fact that the vowels require fewer strokes on average. However, the KSPC of the proposed layout is higher than that of EZ-Hangul because of the segmentation of the consonants, i.e. having to type two consecutive letters assigned to the same key. In this case a short timeout or a skip key is used to disambiguate the letters. EZ-Hangul does not suffer from segmentation.

Predicted Movement Time Per Character (Fitt's Model) The predicted movement time based on Fitt's model appears to be comparable for the proposed layout and EZ-Hangul and they both outperform Chon-ji-in. This is encouraging and needs to be investigated further through a controlled user study.

Travel Distance Per Character (TDPC)

A limitation of the KSPC metric is that it does not differentiate between composing a letter by pressing a single key three times or by pressing three different accounted for in the Fitt's predicted movement time, it has been included here for an additional comparison point. The proposed layout requires the least travel distance while EZ-Hangul requires the most. This is because for many of the letters EZ-Hangul requires the control keys to be pressed in combination with the other keys.

The metric not presented in this paper but considered in the analysis is the Key Strokes Per Syllable [1]. As mentioned previously due to the nature of the Korean script the syllables can be treated as independent structural units. From the analysis the KSPS and the
KSPC for all three methods were analogous.

\section{Pilot Study}

Three native Koreans (two female and one male) in their early thirties participated in a pilot study to assess the feasibility of the proposed design. Two identified themselves as expert users of Chon-ji-in. The subjects were given twelve training phrases followed by twenty one test phrases categorized as short, medium, and long with 27.1, 41.3, and 65.7 letters on average. Each test trial recorded the keystroke sequence and completion time.

The experimental results show that the actual completion times correlate with the predicted times using Fitt's model, which assumes expert users, with the subjects taking just over 17 seconds longer on average (Figure 11). KSPC error rate analysis [7] indicates that the subjects were able to enter the phrases with little editing (Figure 12). Not shown here is that the edit distance between the entered phrases and the test phrases was small. This needs to be quantified further using the error metrics recently introduced in [3].

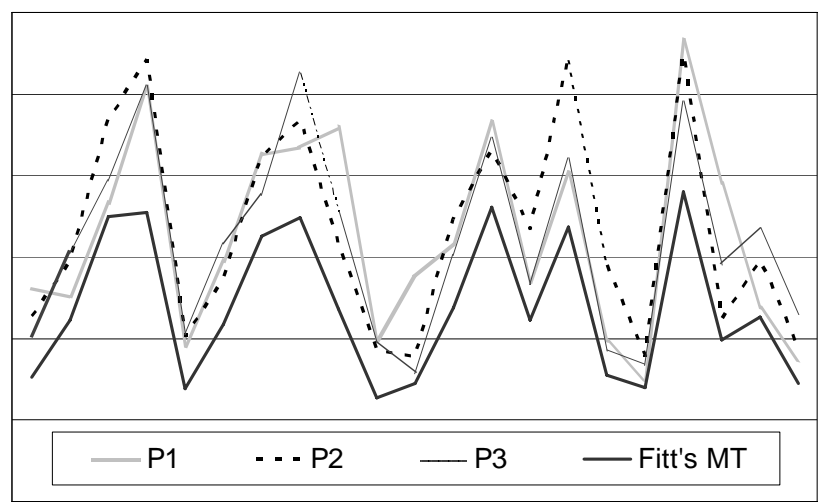

Figure 11. Fitt's predicted time vs. subjects' completion time. 


\begin{tabular}{ccccc}
\hline & mean & std dev & $\max$ & $\min$ \\
\hline P1 & 1.18 & 0.27 & 2.00 & 1.00 \\
\hline P2 & 1.05 & 0.10 & 1.38 & 1.00 \\
\hline P3 & 1.06 & 0.06 & 1.20 & 1.00 \\
\hline
\end{tabular}

Figure 12. KSPC error rate analysis (averaged over 21 trials).

Qualitative feedback indicates that this design affords ease of recognition of the key mappings for vowels.

Considering that this was the first time the subjects were seeing this layout, the quantitative results and the feedback are encouraging.

\section{Conclusion and Future Work}

This paper presented a new keypad layout for Korean text entry for mobile phones. The feasibility of the design was established through a pilot study and further analysis demonstrated that the proposed design compares favorably with two of the predominant Korean text entry methods.

Important questions remain that cannot be answered by the analytical model such as ease of learning and error rate. Future work will consider refinements of the proposed layout based on feedback from the pilot study and will assess the usability and effectiveness of the layout through an observer experiment.

\section{Acknowledgements}

The authors thank the participants in the pilot study.

\section{References}

[1] Doo, M., Lyons, K., and Starner, T. The Korean Twiddler: One-handed chording text entry for Korean mobile phones. Ext. Abstracts CHI 2006, ACM Press (2006), 718-723.

[2] Fitts, P. The information capacity of the human motor system in controlling the amplitude of movement. J ournal of Experimental Psychology: General, 121, 3 (1992), 262-269.

[3] Gong, J. and Tarasewich, P. A new error metric for text entry method evaluation. In Proc. CHI 2006, ACM Press (2006), 471-474.

[4] Kim, S., Kim, G., and Myung, R. Hangul input system's physical interface evaluation model for mobile phone. J ournal of Korean Institute of Industrial Engineers, 28, 2 (2002), 193-200.

[5] MacKenzie, I.S. and Buxton, W. Extending Fitt's law to two-dimensional tasks. In Proc. CHI 1992, ACM Press (1992), 219-226.

[6] Myung, R. Keystroke-level analysis of Korean text entry methods on mobile phones. International J ournal of Human-Computer Studies, 60, (2004), 545-563.

[7] Soukoreff, R.W. and MacKenzie, I.S. Metrics for text entry research: An evaluation of MSD and KSPC, and a new unified error metric. In Proc. CHI 2003, ACM Press (2003), 113-120.

[8] The world of Korean classics. http://gojun.knu.ac.kr. 